\title{
WHAT DOES “REAL-TIME” PROJECT CONTROL REALLY MEAN?
}

\author{
Shabtai Isaac \\ Ben-Gurion University of the Negev, Beer-Sheva, Israel
}

\begin{abstract}
It is proposed that the application of real-time monitoring technologies requires an integration and mutual adjustment of planning and control processes as well, for the automation to be effective. An approach for integrating planning, monitoring and control is presented, and demonstrated in examples of schedule and safety control. Similar solutions can also be developed in other domains. The introduction of automated monitoring technologies can thus lead to future research that will revolutionize planning and control processes, and construction management in general.
\end{abstract}

\section{Introduction}

The need for real-time monitoring and control of construction projects stems from the fact that nearly all projects display significant discrepancies between their actual execution and their plans. The monitoring of large projects is currently a challenge when carried out manually (Isaac and Navon 2014). Consequently, the updating of construction plans according to the actual progress in projects is often carried out intermittently, and largely post factum. Automation presents a possible solution to this problem, by enabling real-time project monitoring. A large number of studies have consequently focused on the application of novel technologies, such as laser scanning, computer vision technology, radio frequency identification, and others, to enable real-time automated monitoring and control (Yang et al. 2015; Omar and Nehdi 2016).

Significant progress has thus been achieved in the testing of automated monitoring technologies. But challenges can still be expected in their application in construction projects. So far, these studies have typically focused on the use of technologies to collect and process large amounts of data, under the assumption that this information can be related to existing construction plans, and utilized for control purposes. They have implicitly been based on a decomposition of project management into a set of distinct and relatively independent processes, which include project planning, monitoring and control. However, manual monitoring cannot be expected to be simply substituted with automated monitoring without requiring the adjustment of other related processes in construction as well.
Past experience from other domains in which automation has been introduced, such as flight deck systems and operating rooms, indicates that the integration of automated components in a management system is not trivial. Sarter et al. (1997) have named this phenomenon the "substitution myth", stating that "[t]he assumption was that new automation can be substituted for human action without any larger impact on the system in which that action or task occurs, except on output", but that "investigations of the impact of new technology have shown that these assumptions are not tenable". The result of this misconception has been that the expected benefits of automation were often only partially achieved, and the workload for human operators only partially relieved, due to the introduction of new tasks.

A number of challenges can be expected in the actual application of automated systems:

- A challenge of incorporating frequent changes that are made in the plan, and novel, unexpected circumstances, within the automated system (Dekker et al. 2002).

- A lack of clarity as to when automated systems should initiate communication with human operators and require their intervention (Sarter et al. 1997).

- Problems of data overload, especially on those occasions when something has gone wrong, which requires human operators to quickly digest and interpret large amounts of relevant data as the events in the project unfold (Woods 1996).

The problem of data overload can be particularly vexing. Automated systems have shown a tendency to increase in scope over time, especially in complex environments, and to become more and more demanding in terms of administrative burdens. There is always a danger of crossing a line, whereupon the system turns from an aid into a hindrance, which users will try to avoid since it takes up too much of their time. With an automated "real-time control" system that is based on large amounts of monitoring data, and may contain practically limitless inputs, this danger becomes particularly relevant. This paper discusses what can be done to avoid such a risk, and how the opportunities of automation can best be realized, by adjusting all the relevant project management processes. 


\section{Proposed approach to real-time project control}

A clear definition of the exact meaning of the terms project planning, project monitoring and project control could be a helpful first step in addressing the challenge that has been presented in the previous section, since these terms are often inconsistently used. The term "project control", for example, can be used to refer either to the execution of corrective actions to minimize deviations (e.g. (Al-Jibouri 2003, Hegazy and Petzold 2003, Lee, Pena-Mora and Park 2006)) or to the process of both identifying deviations and addressing them with corrective actions (e.g. (Diekmann and Al-Tabtabai 1992, Ballard and Howell 1998, Love et al. 2002)). In this paper the different terms are used as follows:

- $\quad$ Project planning - the allocation of resources (such as time, space and manpower) required to execute construction activities.

- $\quad$ Project monitoring - the identification of deviations from the planned budget, schedule and owner requirements in the actual execution of the project.

- $\quad$ Project control - the execution of actions necessary to correct or minimize significant deviations identified in the monitoring process.

These processes are interdependent, and will therefore affect each other (Figure 1). Project monitoring involves a comparison of data that is collected on the construction site with the planning data, an identification of any discrepancies between the two datasets, and an updating of the plan in order to analyze the implications of those discrepancies that have been identified.

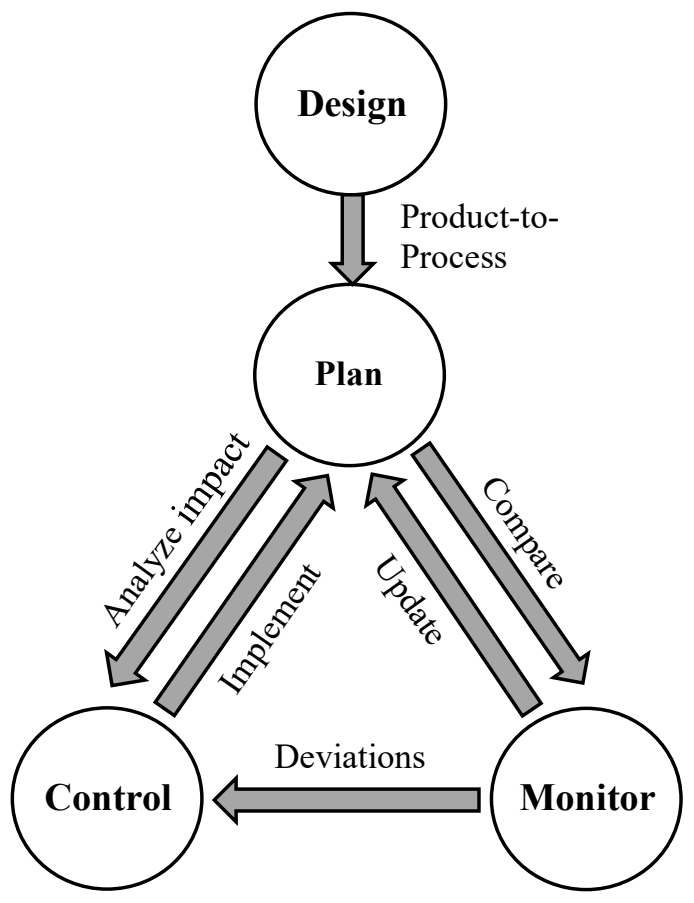

Figure 1: The relations between project planning, monitoring and control
Project control involves the proposal of actions to cope with deviations that have been identified in the monitoring process, and to prevent a further deterioration. This requires an analysis of proposed actions to identify their impact on the project, and an implementation in the project plan of approved actions that are identified as having the desired impact.

The execution of these processes may not be straightforward, since the significance of a deviation may eventually depend on both corrective measures taken by the project management team, such as rescheduling tasks, requesting design changes and allocating additional resources, and on knock-on effects that may cause additional deviations in the future. The team has to determine that such actions will not have a negative indirect impact on the entire project, which could eventually cause a further deterioration (e.g. when additional tasks are executed simultaneously to overcome a delay, and this causes conflicts which result in additional downstream work and further delays).

Given the interdependencies between the automated monitoring process and the largely manual planning and control processes, an integration and mutual adjustment of all three processes is required for the automation to be effective. Thus, existing planning methods will have to be adjusted with the adoption of novel monitoring technologies, in the same way that the application of these technologies will have to take into account those planning practices. In particular, this will require a synchronization of the planning and real-time monitoring methods, which currently address completely different time-scales (Isaac and Navon 2014). For example, schedules are defined, and ideally (but rarely in practice) updated on a daily basis, whereas monitoring technologies can provide data at intervals of minutes or even seconds. In addition, monitoring technologies address projects at a much more detailed scale than do current schedules (Han et al. 2015). For example, an activity such as "Form/Rebar/Pour/Strip Concrete Walls" in the schedule contains, in practice, a number of different sub-activities related to different components (walls), each of which need to be separately defined and addressed by automated monitoring methods.

This paper proposes that the integration of planning, monitoring and control processes requires the following systematic analysis:

- What information is required for control purposes?

- What data can realistically be provided through automated monitoring technologies?

- How can this data be processed in real-time to provide useful information?

- How should project planning and control processes be accordingly revised?

In the following sections, examples of the implementation of such an analysis and integration is briefly described, concerning schedule and safety control. 


\section{Example 1 - Schedule Control}

Automated progress monitoring solutions have not been integrated up till now with innovative project-wide planning and scheduling methods. Instead, they have largely relied on schedules defined with the conventional Critical Path Method (CPM) (e.g. Turkan et al. 2012). However, it is almost impossible to correctly revise a schedule according to monitoring data while using the existing CPM. The main causes for this are the following:

- The CPM currently assumes that the production rate in construction activities is constant, resulting in linear progress functions. In practice, actual production rates fluctuate, due to variations in the project's design (affecting the complexity of the work), on the construction site (e.g. soil conditions), or in the allocation of resources (e.g. the introduction of additional equipment).

- With the current CPM, activities can only be controlled at their end-points (Start or Finish), since the traditional precedence relations connect activities through their end-points alone. This can lead to instances in which deviations occur while an activity is being executed, after it has started but before it has finished, without the CPM providing any means to revise the schedule accordingly. This can happen despite the fact that such a deviation may potentially affect other activities that are executed in parallel, and the project as a whole.

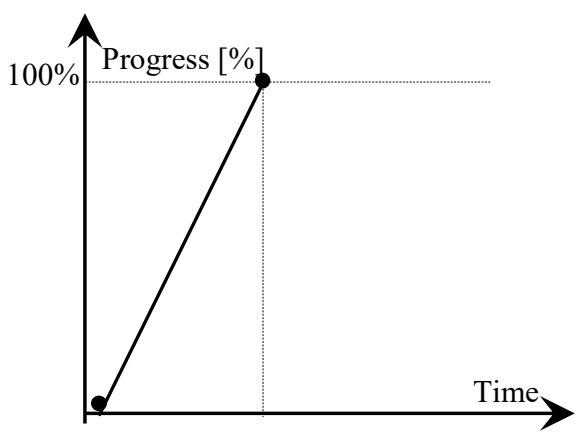

a) Ideal production rate

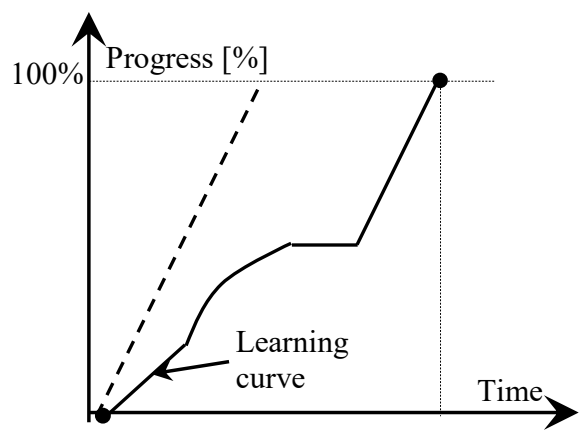

c) Learning curve

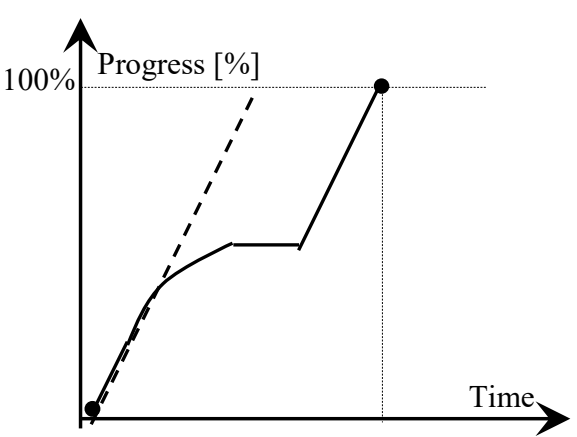

b) Adjustment of production rate for specific segments

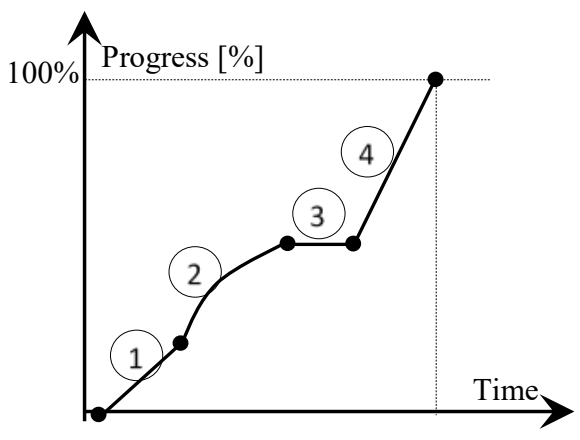

d) Definition of control points

Figure 2. Assessment of production rates and definition of control points 
It is proposed that a key to ensuring effective automated schedule control processes is the correct definition of control points for each activity in the schedule (Figure 2). The progress that has been made during the execution of the activity can be monitored at those points, and the future progress can be predicted. This is especially important given the expected variability in the actual progress when construction activities are carried out. Recent innovations of the CPM provide opportunities to overcome these problems, in particular the development of point-to-point relationships between activities (Hajdu and Isaac 2017). Such point-to-point relationships allow a discrete monitoring of a specific number of control points during the execution of the activity. The automated monitoring can thus be scheduled to be carried out at those points.

The definition of control points for an activity can be carried out in the following steps:

a. Definition of an "ideal" production rate of an activity according to its relevant attributes (e.g. allocated resources and their productivity) (Figure 2a).

b. Adjustment of the "ideal" production rate for specific segments in consideration of activitylevel attributes that may change the amount of work in those segments (e.g. resource allocation, soil conditions, complexity of work) (Figure 2b).

c. Further adjustment of the production rate for initial segments of the activity according to an assessed learning curve (Figure 2c).

d. Definition of control points (Figure 2d):

i. When production rates change

ii. When a sub-activity is completed

iii. According to control points in related activities

iv. At certain intervals if required

Once the activities, composed of segments with control points at their ends, have been modeled as piecewise functions, relationships are defined between the control points of different dependent and overlapping activities (Figure 3). Corresponding control points may have to be defined for overlapping activities, and this may consequently require the definition of additional points. For example, an additional control point is defined for the predecessor activity in Figure 3 in order to link it through a point-to-point relationship with the successor activity. The relationship is connected to a control point where a new segment of the successor activity starts, with a different production rate.

Once the actual progress that was made at the control points has been the recorded in the schedule according to the monitoring data, the remaining work can be updated. In order to analyze the implications of a changing production rate that was observed for an activity on the remainder of that activity and on the production rates of successor activities, there is a need to identify the attributes causing the change in the completed segments of the activity. For example, activity-level attributes (e.g. unexpected soil conditions) are differentiated from project-level activities (e.g. low-skilled manpower).

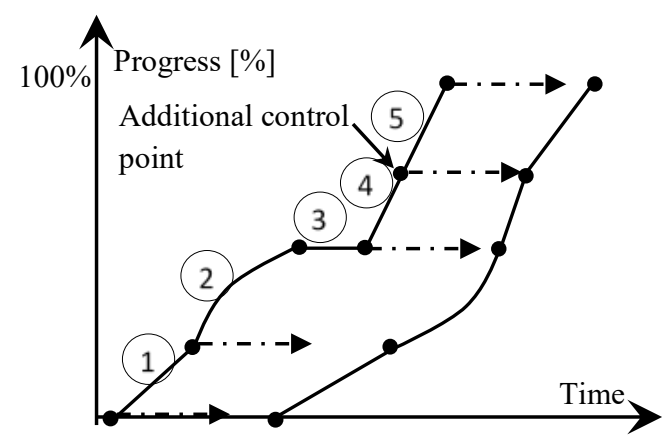

Figure 3. Definition of point-to-point relationships

Based on this, a prediction can be made whether the remaining segments and the successor activities will also be affected by the same attributes:

- If the attributes causing the observed change are relevant only for the completed segments, then the production rates of the remaining segments and of the successor activities don't need to be changed.

- If the attributes are activity-specific, then the production rates of the remaining segments may need to be changed, but not those of the successor activities.

- If the attributes affect the entire work package or project, then the rates of all remaining work may need to be changed.

Once the remaining time for ongoing and planned activities has been defined, additional control actions can be taken in order to reduce deviations from the planned schedule:

1. An activity can be stopped and delayed to satisfy the relationship.

2. Resources can be added or reallocated to change production rates for specific segments of activities.

3. The relationship can be adjusted in case neither one of the points on the activities has been reached yet.

4. The schedule can be manually adjusted.

The proposed approach can support the synchronization, according to the predefined control points on activities, of planning, monitoring and control. This will allow them to be carried out at the same required time intervals, through a combination of both automated and manual processes.

\section{Example 2 - Safety Control}

The previous section presented an approach that could be suitable for progress monitoring and the updating of the project schedule. While that example put more of an emphasis on the need to adjust the planning and integrate it with the automated monitoring process, other research by the author has focused on the adjustment of control 
processes as well, in the context of automated safety monitoring with real-time location tracking of workers (Isaac and Edrei 2016, Edrei and Isaac 2017).

Previous studies on automated safety monitoring usually adopted a deterministic planning and control approach involving the "virtual fencing" of high-risk areas (resembling existing approaches with physical fences), and alerts whenever an unauthorized worker penetrated into such a high-risk area. This planning approach might not however be suitable for real-time monitoring, which should support proactive alerts. Instead, an alert should be provided sufficiently early before an accident occurs, when it is still possible to carry out a preventative action. In order to be able to do so, there is a need to detect those instances in which a worker has increased his exposure to hazards because he is moving nearer to a high risk area (Figure 4), or because he is exposing himself for too long a time to a low or moderate-level risk factor (such as noise).

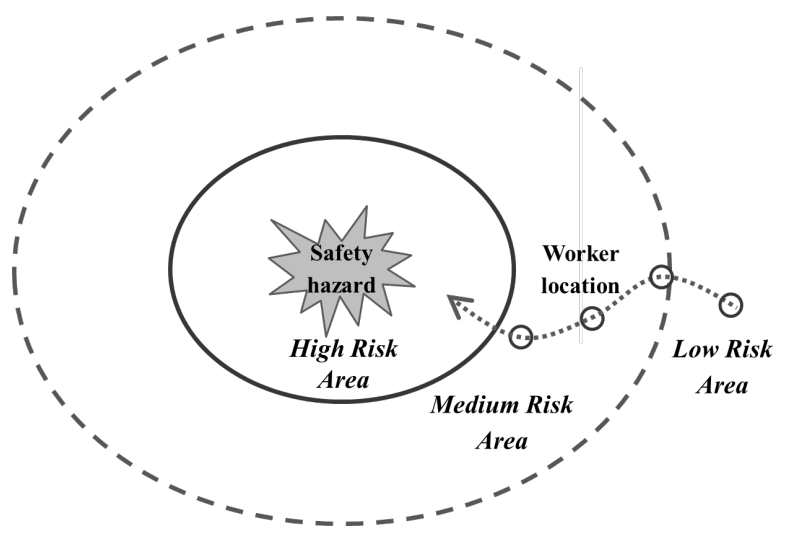

Figure 4. Tracking a worker approaching a safety hazard

In Isaac and Edrei (2016), a statistical model was presented that supports a more dynamic form of safety planning and control, which better utilizes the real-time tracking data. The model relied on data that was received from a wi-fi-based location tracking system. The new planning approach included not only the definition of high-risk areas which are off-limits for all non-authorized workers, but also of medium-risk areas, in which a certain degree of risk exposure is tolerated. While medium-risk areas may appear to be free of critical or immediate hazards, a presence in such an area may harm a worker after some time, or indicate that he is moving too close to a high risk area.

The proposed model provides statistical alerts when the risk exposure of a worker has exceeded tolerable levels. This is enabled through the application of a number of statistical rules that detect an increase in the exposure to hazards, and provide an alert before a critical exposure occurs. To enable this, statistical safety zones are defined in the model. Each statistical zone is related to a risk area on the site, and reflects the expected probability of a worker being located in this risk area. The statistical zones are based on a predefined maximum allowable exposure to the safety risk that has been identified. In other words, an area in which the exposure of workers to a safety risk is higher, should have a correspondingly lower probability that the worker will be located in it. In this way, the model can be used to control the movement of workers on the site, and to proactively impose certain limits to their risk exposure.

The planning and control process that was developed can prevent potential accidents from occurring, without unnecessarily affecting the efficiency of the activities carried out on site. On the one hand, transgressions of medium-risk areas on the site are likely to be frequent, implying that the creation of alerts whenever such a transgression occurs would excessively restrict the workers, and probably lead to them ignoring the alerts. On the other hand, the new process is not limited to providing alerts once a worker has already penetrated a high-risk area, which can have only a limited impact in terms of preventing accidents and injuries. The new process can thus ensure the health and safety of workers, and at the same time the efficiency of the construction work, by restricting the size of the areas that are defined as being of high risk, and therefore off-limits for most workers on site.

The new process allows site managers to control the exposure of construction workers to safety risks that accumulate and change over time, providing alerts when workers approached risk factors too closely, and ensuring their health by preventing excessive exposure over time to such factors. This required, however, a novel planning framework based on the definition of medium-risk areas, and a novel control process based on statistical rules.

\section{Conclusions}

The integration of automated monitoring with planning and control processes is crucial in order to fully exploit the benefits of the automated technologies. Such an integration will require mutual adjustments of all these processes. It has to be applied in a wide range of domains, each involving distinct requirements.

Two examples were briefly described of the implementation of such an integration, for schedule and safety control. Similar solutions can also be developed in other domains, such as quality control and cost management. By requiring the adjustment of conventional planning and control processes, the introduction of automated monitoring technologies can therefore be seen as an opportunity for future research to revolutionize those processes as well, and thus the practice of construction management in general.

\section{References}

Al-Jibouri, S H (2003) Monitoring systems and their effectiveness for project cost control in construction. International Journal of Project Management, 21(2), 145-54.

Ballard, G and Howell, G (1998) Shielding production: Essential step in production control. Journal of Construction Engineering and Management, 124(1), 
11-7.

Dekker, S. W., \& Woods, D. D. (2002). MABA-MABA or abracadabra? Progress on human-automation coordination. Cognition, Technology \& Work, 4(4), 240244.

Diekmann, J E and Al-Tabtabai, H (1992) Knowledgebased approach to construction project control. International Journal of Project Management, 10(1), 23-30.

Han, K. K., Cline, D., \& Golparvar-Fard, M. (2015). Formalized knowledge of construction sequencing for visual monitoring of work-in-progress via incomplete point clouds and low-LoD 4D BIMs. Advanced Engineering Informatics, 29(4), 889-901.

Hegazy, T and Petzold, K (2003) Genetic optimization for dynamic project control. Journal of Construction Engineering and Management, 129(4), 396-404.

Isaac, S., \& Edrei, T. (2016). A statistical model for dynamic safety risk control on construction sites. Automation in Construction, 63, 66-78.

Edrei, T., \& Isaac, S. (2017). Construction site safety control with medium-accuracy location data. Journal of Civil Engineering and Management, 23(3), 384392.

Hajdu, M., \& Isaac, S. (2016). Sixty years of project planning: history and future. Organization, Technology and Management in Construction: an International Journal, 8(1), 1499-1510.

Isaac, S., \& Navon, R. (2014). Can project monitoring and control be fully automated?. Construction management and economics, 32(6), 495-505.

Lee, S, Pena-Mora, F and Park, M (2006) Reliability and stability buffering approach: Focusing on the issues of errors and changes in concurrent design and construction projects. Journal of Construction Engineering and Management, ASCE, 132(5), 452-64.

Love, P E D, Holt, G D, Shen, L Y, Li, H and Irani, Z (2002) Using systems dynamics to better understand change and rework in construction project management systems. International Journal of Project Management, 20, 425-36.

Omar, T., \& Nehdi, M. L. (2016). Data acquisition technologies for construction progress tracking. Automation in Construction, 70, 143-155.

Sarter, N.B., Woods, D.D. and Billings, C.E. (1997). Automation surprises. Handbook of human factors and ergonomics, 2, pp.1926-1943.

Turkan, Y., Bosche, F., Haas, C. T., \& Haas, R. (2012). Automated progress tracking using 4D schedule and 3D sensing technologies. Automation in Construction, 22, 414-421.

Woods, D. D. (1996). Decomposing automation: Apparent simplicity, real complexity. Automation and human performance: Theory and applications, 3-17.

Yang, J., Park, M. W., Vela, P. A., \& Golparvar-Fard, M. (2015). Construction performance monitoring via still images, time-lapse photos, and video streams: Now, tomorrow, and the future. Advanced Engineering Informatics, 29(2), 211-224. 